\title{
Adult Case of Atlantoaxial Rotatory Fixation Treated with In Situ Fixation Using an Unusual Posterior Instrumentation Construct
}

\author{
Yasuyoshi Miyao, ${ }^{1}$ Manabu Sasaki, ${ }^{2}$ Masao Umegaki, ${ }^{2}$ and Kazuo Yonenobu ${ }^{3}$
}

\begin{abstract}
Atlantoaxial rotatory fixation (AARF) occurs commonly in children who have undergone trauma. It is usually corrected with conservative therapy. In this report, however, the patient was an adult with AARF who was treated surgically. A 64-year-old woman presented with a 1-year history of spastic gait and hand clumsiness. Imaging studies revealed the presence of AARF, os odontoideum, and severe spinal cord compression at that spinal level. As the AARF had not been responded to head traction with a halo device, we decided to treat the patient with in situ posterior fixation. Because the rigid dislocation was present between the atlas and the axis, we were forced to make an unusual instrumentation construct. Neurological symptoms other than hand numbness diminished after the surgery, and arthrodesis was obtained between the occiput and the axis. It should be noted that surgical planning for posterior instrumentation construct is required when rigid AARF is treated surgically.
\end{abstract}

Keywords: atlantoaxial rotatory fixation, os odontoideum, surgical treatment, posterior instrumentation

\section{Introduction}

Atlantoaxial rotatory fixation (AARF), which is common in children, is caused by trauma and is usually corrected with conservative therapy. ${ }^{1-3)}$ Delay of the diagnosis, however, makes it difficult to correct it conservatively. ${ }^{4-6)}$ In these cases, intraoperative reduction and posterior fixation is performed. In adults, AARF is rarely caused by trauma and can usually be corrected by traction using a halo device. ${ }^{5,6}$ Owing to the rarity of adult cases, however, there are few descriptions of the operative method for AARF. We describe an adult case of AARF with os odontoideum who was treated by $\mathrm{C} 1$ laminectomy and in situ occiput- $\mathrm{C} 2$ fixation.

\section{Case Report}

A 64-year-old woman had suffered from unsteady gait, hand clumsiness, and numbness since October 2013. She initially consulted a neurologist, who diagnosed cervical myelopathy. When she was introduced to our department in

${ }^{1}$ Department of Neurosurgery, Suita Municipal Hospital, Suita, Osaka, Japan

${ }^{2}$ Department of Neurosurgery, Iseikai Hospital, Osaka, Osaka, Japan ${ }^{3}$ Graduate School of Health Care Sciences, Jikei Institute, Osaka, Osaka, Japan

Received: November 12, 2016; Accepted: December 2, 2016 Online June 9, 2017
February 2014, she could not stand without support. The Romberg sign and Hoffman reflexes were positive. The 10-s grasp-and-release test was performed 10 times for the right hand and 11 times for the left. The manual muscle test revealed weakness of 3-4/5 in the extremities.

Computed tomography (CT) scans revealed AARF and os odontoideum (Fig. 1). The right synovial joint was intact and worked as an axis of rotation (Fig. 1B). The left superior articular process of the axis was posteriorly dislocated relative to the left inferior articular process of the atlas (Figs. 1C-1F). Magnetic resonance imaging (MRI) revealed severe compression of the spinal cord between the $\mathrm{C} 1$ lamina and the dens (Fig. 2A). T2-weighted images revealed intramedullary high-intensity changes at this level (Figs. 2B-2D), suggesting that the myelopathy was caused by spinal canal stenosis due to AARF and its instability. As AARF had not been corrected by halo traction, we decided to treat it surgically. Before the surgery, which was performed in May 2014, we checked the occiput-C2 angle to ensure that it was suitable for easy swallowing under external halo fixation.

After introduction of general anesthesia, the patient was positioned prone with her neck fixed in the occiput-C2 angle that had been determined preoperatively. Initially, spinal cord decompression was achieved by laminectomy of the atlas, followed by placement of the posterior instrumentation (Fig. 3). The Y-shaped posterior cranial plate was fixed with screws in the midline of the occiput, and pedicle screws were inserted bilaterally at the $\mathrm{C} 2$ and $\mathrm{C} 3$ levels under the guidance of a spinal navigator. We did not attempt correction during the operation because we considered it dangerous due to the rigidity of the rotatory dislocation. Connection of the rod and screws was quite difficult on the right side owing to left-lateral dislocation of the occiput relative to the axis. Half of the transverse connector was therefore utilized for the connection between the right-side pedicle screws and the rod fixed on the cranial plate (Fig. 3A). After posterior instrumentation was complete, a bone strut harvested from the left iliac crest was grafted between the occiput and the axis (Fig. 3B).

Postoperatively, the neurological symptoms diminished, and she soon became ambulatory without support. The 10-s grasp-and-release test improved 25-fold in the right hand and 20 -fold in the left hand. The chief residual symptom was numbness in both hands. Plain radiography revealed rigid fixation using an unusual posterior instrumentation construct (Figs. 4A and 4B). Postoperative MRI showed that the spinal cord was released at the C1-2 level (Fig. 4C). A CT scan at the 2-year follow-up showed that arthrodesis was obtained between the occiput and the axis (Fig. 4D). 

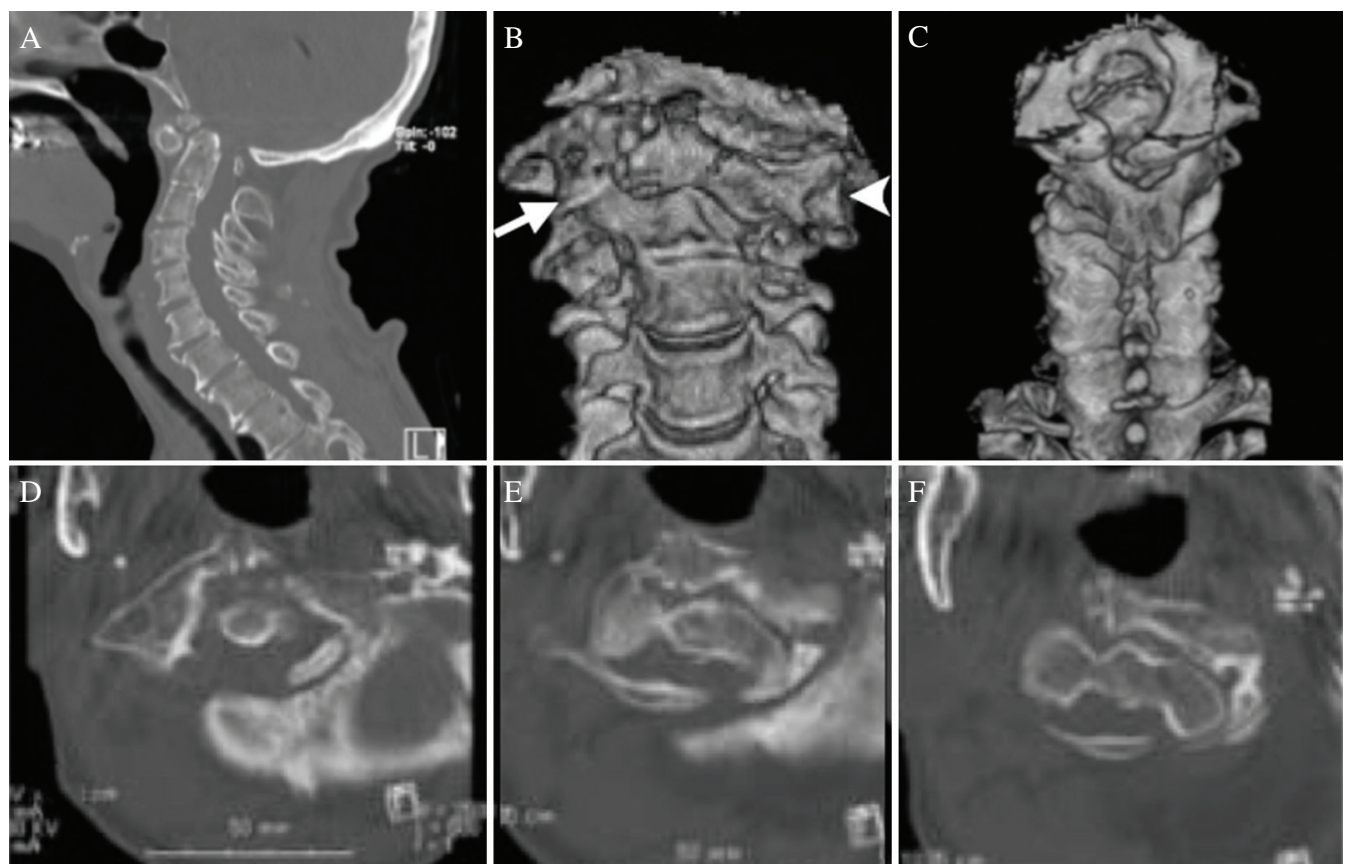

Fig. 1 Preoperative computed tomography (CT) scan shows os odontoideum (A) and atlantoaxial rotatory dislocation (B-F). The right synovial joint was intact (arrow) and worked as an axis of rotation. The left superior articular process (SAP) of the axis was dislocated posteriorly relative to the left inferior articular process of the atlas $(\mathrm{C}-\mathrm{F})$. $(\mathrm{B}, \mathrm{C})$ The posteriorly dislocated SAP of the axis (arrowhead) is observed on three-dimensional CT.
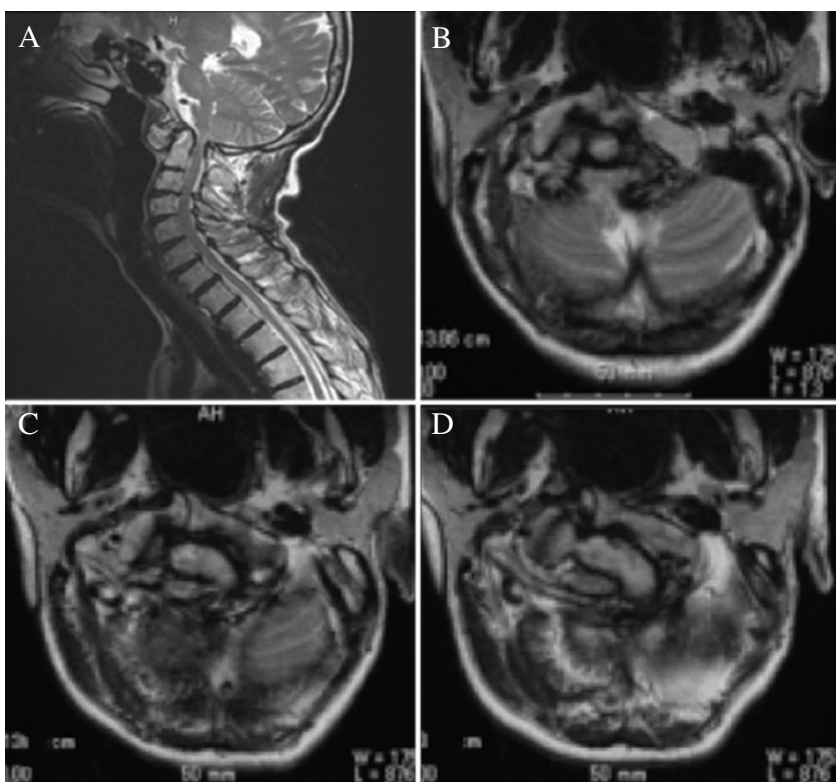

Fig. 2 Preoperative magnetic resonance imaging (MRI) shows spinal cord compression between the dens and the posterior arch of the atlas (A) as well as intramedullary high-intensity changes at that spinal level (B-D).

\section{Discussion}

AARF was first described by Fielding and Hawkins as persistent subluxation of the atlantoaxial joint caused by torticollis in children. ${ }^{1)}$ It exhibits the cock-robin deformity, which is caused by rotating the head to one side while flexing it laterally to the other side. Its etiology is not clear,

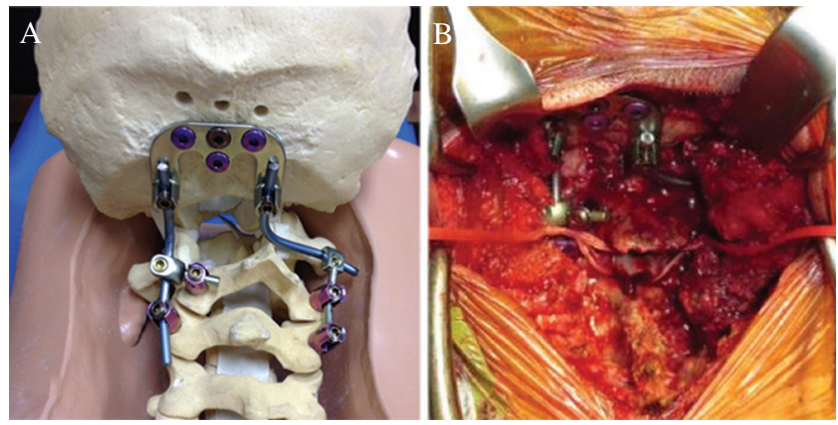

Fig. 3 A unique posterior instrumentation construct created in the present case. Connection of the rod and the screws was difficult on the right side owing to left-lateral dislocation of the occiput relative to the axis. Half of the transverse connector was used to connect the right-side pedicle screws and the rod fixed on the cranial plate (A). A bone strut harvested from the left iliac crest was grafted between the occiput and the axis (B).

but it could be caused by trauma, ${ }^{7)}$ infection, or timeconsuming otolaryngological surgery. Trauma or infection induces neck muscle tension, increases synovial fluid, extends ligaments, and finally induces subluxation. ${ }^{8)}$ AARF is frequent in children because of their undeveloped bone structures, loose and extreme rotation, and horizontalization of the atlantoaxial joint. ${ }^{910)}$ AARF rarely causes symptoms in adults. Some previous reports indicated that AARF may be caused in adults by ankylosing spondylitis, ${ }^{11)}$ metastasis ${ }^{12)}$ and eosinophilic granuloma. ${ }^{13)}$ To the best of our knowledge, this is the first report on AARF due to os odontoideum. 

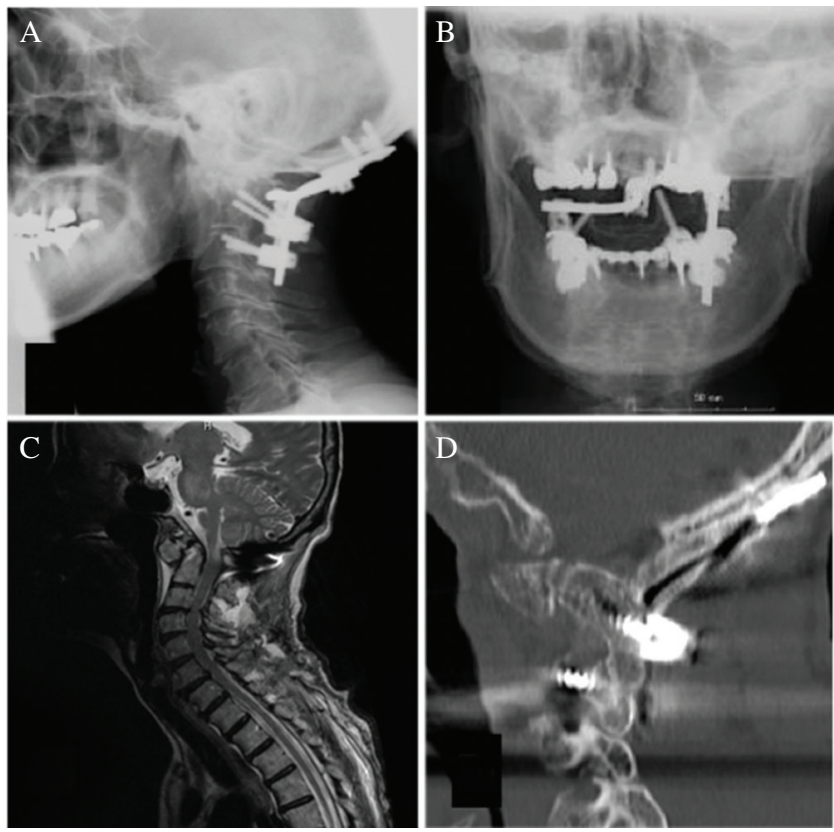

Fig. 4 Postoperatively, plain radiography showed in situ fixation with the unique construct for posterior fixation from the occiput to the $\mathrm{C} 3$ level (A, B). MRI shows that the spinal cord was released from compression at the C1-2 level (C). CT scans at the 2-year follow-up show that arthrodesis was obtained between the occiput and the axis.

Multiplanar reconstruction CT is useful for diagnosing AARF. ${ }^{14)}$ It can clearly demonstrate deformity of the facet joints. The presence of lateral inclination is thought to be a sign of a poor prognosis with conservative treatment. ${ }^{15)}$ Degeneration of the atlantoaxial synovial joint is also detectable with arthrography. ${ }^{9}$

The efficacy of the treatment depends on an early definitive diagnosis. If AARF is diagnosed within a month after its occurrence, it can usually be corrected with conservative therapy, such as traction and external fixation with a brace. ${ }^{6,16)}$ A previous report suggested that AARF diagnosed more than 4 weeks after the causative incident is potentially treatable with conservative treatment if MRI shows patency of the transverse ligament. ${ }^{17)}$ Conversely, a delayed diagnosis usually results in a poor outcome with conservative therapy. ${ }^{4,718)}$ As reported previously, AARF can become chronic when it is caused by fibrous tissue degeneration and osteofusion. $^{15,19)}$

If recurrent or intractable symptoms are present after conservative therapy, surgical treatment should be considered. According to previous reports, surgical procedures for AARF often include intraoperative reduction and fusion via a posterior, anterior, or lateral approach. ${ }^{18,20-23)}$ Most of the patients in those reports were children, and the rotatory dislocation of the synovial joints might have been relatively easy to correct.

There is a potential risk, however, of spinal cord injury during corrective procedures for rigid AARF such as in the present case. Therefore, we decided to perform neural decompression with laminectomy of the atlas and in situ fixation between the occiput and the axis. We often utilize a Y-shaped occipital plate fixed with screws that penetrate the middle part of the occipital bone for rigid stabilization. In this case, however, it was difficult to construct a device with a conventional polyaxial screw and rod system because of the rotatory dislocation between the occiput and the axis. Therefore, we uniquely utilized half of the transverse connector as a joint between the screws and the rod on the right side. Arthrodesis was obtained with this construct. It should be noted that an unusual construct of the posterior instrumentation may be required when rigid AARF is treated surgically.

\section{Conflicts of Interest Disclosure}

None of the authors has any conflicts of interest to disclosure.

\section{References}

1) Fielding JW, Hawkins RJ: Atlanto-axial rotatory fixation. (Fixed rotatory subluxation of the atlanto-axial joint). J Bone Joint Surg Am 59: 37-44, 1977

2) Martinez-Lage JF, Martinez Perez M, Fernandez Cornejo V, Poza M: Atlanto-axial rotatory subluxation in children: early management. Acta Neurochir (Wien) 143: 1223-1228, 2001

3) Subach BR, McLaughlin MR, Albright AL, Pollack IF: Current management of pediatric atlantoaxial rotatory subluxation. Spine 23: 21742179, 1998

4) Tarantino R, Donnarumma P, Marotta N, Missori P, Viozzi I, Landi A, Delfini R: Atlanto axial rotatory dislocation in adults: a rare complication of an epileptic seizure_case report. Neurol Med Chir (Tokyo) 54: 413-416, 2014

5) Escobar LEM, Osterhoff G, Ossendorf C, Wanner GA, Simmen H-P, Werner CM: Traumatic atlantoaxial rotatory subluxation in an adolescent: a case report. J Medical Case Reports 6: 27, 2012

6) Jeon SW, Jeong JH, Moon SM, Choi SK: Atlantoaxial rotatory fixation in adults patient. J Kor Neurosurg Soc 45: 246-248, 2009

7) Crook TB, Eynon CA: Traumatic atlantoaxial rotatory subluxation. Emerg Med J 22: 671-672, 2005

8) Wortzman G, Dewar FP: Rotary fixation of the atlantoaxial joint: Rotational atlantoaxial subluxation. Radiology 90: 479-487, 1968

9) Ono K, Yonenobu K, Fuji T, Okada K: Atlantoaxial rotatory fixation. Radiographic study of its mechanism. Spine 10: 602-608, 1985

10) Crossman JE, Thompson D, Hayward RD, Ransford AO, Crockard HA: Recurrent atlantoaxial rotatory fixation in children: a rare complication of a rare condition. Report of four cases. J Neurosurg 100: 307-311, 2004

11) Leventhal MR, Maguire JK, Christian CA: Atlantoaxial rotary subluxation in ankylosing spondylitis. A case report. Spine 15: 1374-1376, 1990

12) White GM, Healy WL: Tumor-associated atlanto-axial rotatory fixation. A case report. Spine 12: 406-408, 1987

13) Marar BC, Balachandran N: Non-traumatic atlanto-axial dislocation in children. Clin Orthop Relat Res 220-226, 1973

14) Been HD, Kerkhoffs GM, Maas M: Suspected atlantoaxial rotatory fixation-subluxation: the value of multidetector computed tomography scanning under general anesthesia. Spine 32: E163-E167, 2007

15) Ishii K, Chiba K, Maruiwa H, Nakamura M, Matsumoto M, Toyama Y: Pathognomonic radiological signs for predicting prognosis in patients with chronic atlantoaxial rotatory fixation. J Neurosurg Spine 5: 385-391, 2006

16) Han ZM, Nagao N, Sakakibara T, Akeda K, Matsubara T, Sudo A, Kasai Y: Adult traumatic atlantoaxial rotatory fixation: a case report. Case Reports Orthoped 2014: 2014, 593621

17) Castel E, Benazet JP, Samaha C, Charlot N, Morin O, Saillant G: Delayed closed reduction of rotatory atlantoaxial dislocation in an adult. Eur Spine J 10: 449-453, 2001

18) Crossman JE, David K, Hayward R, Crockard HA: Open reduction of pediatric atlantoaxial rotatory fixation: long-term outcome study with functional measurements. J Neurosurg 100: 235-240, 2004 
19) Pang D, Li V: Atlantoaxial rotatory fixation: part 3-a prospective study of the clinical manifestation, diagnosis, management, and outcome of children with alantoaxial rotatory fixation. Neurosurgery 57: 954-972; discussion 954-972, 2005

20) Belen D, Simsek S, Yigitkanli K, Bavbek M: Internal reduction established by occiput-C2 pedicle polyaxial screw stabilization in pediatric atlantoaxial rotatory fixation. Pediatr Neurosurg 42: 328-332, 2006
21) Stulik J, Vyskocil T, Sebesta P, Kryl J: Atlantoaxial fixation using the polyaxial screw-rod system. Eur Spine J 16: 479-484, 2007

22) Goel A, Shah A: Atlantoaxial facet rocking: treatment of facet manipulation and fixation. Experience of 14 cases. J Neurosurg Spine 14: 3-9, 2011

23) Govender S, Kumar KP: Staged reduction and stabilisation in chronic atlantoaxial rotatory fixation. J Bone Joint Surg Br 84: 727-731, 2002 